\title{
Curved Casimir Operators and the BGG Machinery ${ }^{\star}$
}

\author{
Andreas $\check{C} A P^{\dagger \ddagger}$ and Vladimir SOUČEK ${ }^{\S}$ \\ $\dagger^{\dagger}$ Fakultät für Mathematik, Universität Wien, Nordbergstr. 15, A-1090 Wien, Austria \\ ¥ International Erwin Schrödinger Institute for Mathematical Physics, \\ Boltzmanngasse 9, A-1090 Wien, Austria \\ E-mail: Andreas.Cap@esi.ac.at \\ $\S$ Mathematical Institute, Charles University, Sokolovská 83, Praha, Czech Republic \\ E-mail: soucek@karlin.mff.cuni.cz
}

Received August 24, 2007, in final form November 16, 2007; Published online November 22, 2007

Original article is available at http://www.emis.de/journals/SIGMA/2007/111/

\begin{abstract}
We prove that the Casimir operator acting on sections of a homogeneous vector bundle over a generalized flag manifold naturally extends to an invariant differential operator on arbitrary parabolic geometries. We study some properties of the resulting invariant operators and compute their action on various special types of natural bundles. As a first application, we give a very general construction of splitting operators for parabolic geometries. Then we discuss the curved Casimir operators on differential forms with values in a tractor bundle, which nicely relates to the machinery of BGG sequences. This also gives a nice interpretation of the resolution of a finite dimensional representation by (spaces of smooth vectors in) principal series representations provided by a BGG sequence.
\end{abstract}

Key words: induced representation; parabolic geometry; invariant differential operator; Casimir operator; tractor bundle; BGG sequence

2000 Mathematics Subject Classification: 22E46; 53A40; 53C15; 58J70

Dedicated to the memory of Thomas P. Branson

\section{Introduction}

Let $G$ be a semisimple Lie group and $P \subset G$ a parabolic subgroup. Then to any representation of $P$ one may associate a representation of $G$ by the process of parabolic induction. The induced representations are most naturally defined on spaces of sections of homogeneous vector bundles on the generalized flag manifold $G / P$. As on any representation of $G$, the Casimir operator naturally acts on (the smooth vectors of) any induced representation, and it is easy to see that it acts by a $G$-invariant differential operator.

Starting from examples like conformal structures and CR-structures, it has been realized that manifolds endowed with certain geometric structures can be viewed as "curved analogs" of homogeneous spaces of the form $G / P$. More formally, they can be equivalently described as certain Cartan geometries with homogeneous model $G / P$. These structures are called parabolic geometries of type $(G, P)$ and they have been intensively studied during the last years.

Any representation of $P$ gives rise to a natural vector bundle on all parabolic geometries of type $(G, P)$, and on the homogeneous model, this gives rise to the usual homogeneous vector bundles. The concept of $G$-invariant differential operators between sections of homogeneous vector bundles generalizes to the notion of invariant (or natural) differential operators on parabolic geometries. Questions on such operators are surprisingly subtle and are a major research topic, see e.g. $[9,13,12,6,3,11,10]$.

\footnotetext{
${ }^{\star}$ This paper is a contribution to the Proceedings of the 2007 Midwest Geometry Conference in honor of Thomas P. Branson. The full collection is available at http://www.emis.de/journals/SIGMA/MGC2007.html
} 
The first main result of this article is that the Casimir operator on an induced representation canonically extends to a natural operator on arbitrary parabolic geometries of type $(G, P)$, which we call the curved Casimir operator. This extension is described in terms of the socalled fundamental derivative, which is easily connected to well developed tools for parabolic geometries such as tractor calculus.

Next, we prove that, similarly to the original Casimir operator, the curved analog has strong naturality properties. Using ideas closely related to tractor calculus we derive a simple formula for the curved Casimir operator on an arbitrary natural bundle. This is used to show that the curved Casimir always is an operator of order at most one. On natural bundles induced by irreducible representations of $P$ (which correspond to principal series representations) the curved Casimir acts by a scalar, and we compute this scalar in terms of weights. We sketch how this result can be applied to construct a large number of natural differential operators.

In the last section, we consider the curved Casimir operators on the so-called tractor bundles, see [5], as well as on tractor bundle valued differential forms. On the homogeneous model, tractor bundles correspond to representations induced by restrictions to $P$ of representations of $G$, and they are canonically trivial. In the curved case, however, they need not be trivial and they are important tools, since they admit canonical linear connections. We related the curved Casimir operator to these tractor connections and the induced covariant exterior derivative.

A large number of invariant differential operators on parabolic geometries can be constructed using the machinery of Bernstein-Gelfand-Gelfand (or BGG) sequences, which applies to curved geometries. On the homogeneous model, one obtains resolutions of all finite dimensional irreducible representation of $G$ by invariant differential operators between sections of homogeneous vector bundles. These operators are related by a duality to the homomorphisms of generalized Verma modules in the generalized BGG resolution of the dual of the given finite dimensional representation. BGG sequences were first constructed in [6], and the construction was improved in [3]. Our description of the curved Casimir operator sheds new light on this construction. In particular, we prove that the resolution on the homogeneous model is obtained by restricting the twisted de-Rham resolution to eigenspaces of the Casimir operator.

The interaction between results for the homogeneous model (which often are deduced using representation theory) and results on general curved geometries is one of the basic features of the theory of parabolic geometries. This has led to interesting interactions between people working in representation theory and people working on parabolic geometries, but the communication between the two groups is not always easy. One of the few people active in both communities was Tom Branson, who did a lot to facilitate mutual communication. He died completely unexpectedly in 2006, and the 2007 Midwest Geometry Conference was held in his honor. This article is our contribution to the proceedings of the MGC 2007 conference. Trying to make the article accessible for people from both communities, we have included a bit of review material as well as comparison between the languages and notations which are standardly used in the two fields. We hope that this article will be useful for intensifying the interactions between the two communities.

\section{A curved analog of the Casimir operator}

\subsection{Generalized flag manifolds and induced representations}

A generalized flag manifold is the quotient $G / P$ of a semisimple Lie group $G$ by a parabolic subgroup $P \subset G$. These homogeneous spaces are always compact, and in the case that $G$ is complex, they are the only compact homogeneous spaces of $G$. In representation theory, these spaces play an important role via parabolic induction. Suppose that $W$ is a finite dimensional representation of $P$. Then one forms the homogeneous vector bundle $G \times_{P} W=(G \times W) / P$, 
where $P$ acts from the right on $G \times W$ via $(g, w) \cdot h:=\left(g h, h^{-1} \cdot w\right)$. The $G$-action on $G / P$ naturally lifts to an action on $G \times_{P} W$ by vector bundle homomorphisms. Hence the space $\Gamma\left(G \times_{P} W\right)$ of smooth sections carries a natural representation of $G$, called the representation induced by $W$, defined by $(g \cdot s)(\tilde{g} P)=g \cdot\left(s\left(g^{-1} \tilde{g} P\right)\right)$. Using compactness of $G / P$, this representation can be extended to various completions of $\Gamma\left(G \times{ }_{P} W\right)$, and this construction is one of the basic sources of representations of $G$. If one starts with an irreducible representation of $P$, the resulting representation of $G$ is called a principal series representation.

There is an equivalent description of induced representations which is commonly used in representation theory. The space $\Gamma\left(G \times_{P} W\right)$ can be naturally identified with

$$
C^{\infty}(G, W)^{P}=\left\{f \in C^{\infty}(G, W): f(g h)=h^{-1} \cdot f(g) \quad \forall g \in G, h \in P\right\} .
$$

(This depends only on the fact that $G \times{ }_{P} W$ is associated to the principal bundle $G \rightarrow G / P$.) The correspondence between $s \in \Gamma\left(G \times{ }_{P} W\right)$ and $f \in C^{\infty}(G, W)^{P}$ is characterized by the fact that $s(g P)$ is the equivalence class of $(g, f(g))$. In this picture, the natural left action of $G$ is given by $(g \cdot f)(\tilde{g})=f\left(g^{-1} \tilde{g}\right)$.

In some cases, the $G$-action on $G / P$ admits a direct geometric interpretation. For example, if $G=O(n+1,1)$ and $P \subset G$ is the stabilizer of an isotropic line, then $G / P$ is the $n$-sphere $S^{n}$, and for $n \geq 3$, the action on $G / P$ identifies $G$ with the group of conformal isometries of the round metric on $S^{n}$. These are exactly the diffeomorphisms of $S^{n}$, which only rescale the round metric by a positive smooth function. For $G=S U(n+1,1)$ and $P \subset G$ the stabilizer of a complex isotropic line, one obtains $G / P=S^{2 n+1}$, viewed as a hypersurface in $\mathbb{C}^{n+1}$. The action on $G / P$ identifies $G$ with the group of all CR-diffeomorphisms of $S^{2 n+1}$. These are exactly the diffeomorphisms induced from biholomorphisms of the unit ball.

\subsection{Parabolic geometries}

It is an idea due to Elie Cartan to describe certain geometric structures as "curved analogs" of homogeneous spaces, which represent geometries in the sense of Klein's Erlangen program. An exposition of this approach and several examples can be found in the book [16] and in [4], which is specifically directed towards the parabolic case. The starting point of this approach is that the left actions of the elements of $G$ on a homogeneous space $G / H$ can be characterized as those automorphisms of the $H$-principal bundle $G \rightarrow G / H$ which preserve the left MaurerCartan form on $G$. A curved analog, called a Cartan geometry of type $(G, H)$, is then given by a principal $H$-bundle $\mathcal{G}$ on a manifold $M$ such that $\operatorname{dim}(M)=\operatorname{dim}(G / H)$ and a Cartan connection $\omega \in \Omega^{1}(\mathcal{G}, \mathfrak{g})$, where $\mathfrak{g}$ is the Lie algebra of $G$. The Cartan connection is required to define an isomorphism $T \mathcal{G} \rightarrow \mathcal{G} \times \mathfrak{g}$, to be equivariant with respect to the principal right action of $H$, and to reproduce the generators of fundamental vector fields.

Cartan himself proved that conformal structures in dimension $\geq 3$ and $\mathrm{CR}$ structures in dimension 3 can be equivalently described as Cartan geometries corresponding to the homogeneous spaces of $O(n+1,1)$ respectively $S U(2,1)$ mentioned above. For CR structures in arbitrary dimensions this is a famous result of Chern and Moser, see [8], with an earlier proof due to Tanaka. The fact that the Cartan geometries provide an equivalent description of the underlying structure is a non-trivial theorem in these cases. In pioneering work culminating in [17], N. Tanaka showed that for every semisimple Lie group $G$ and parabolic subgroup $P \subset G$, Cartan geometries of type $(G, P)$ (which satisfy the additional conditions of regularity and normality) are equivalent encodings of certain underlying structures. The description of these underlying structures has been later simplified considerably, see [4]. The geometric structures obtained in this way have been named parabolic geometries and have been intensively studied during the last years. Apart from conformal and CR structures, also projective and quaternionic structures, path geometries, quaternionic contact structures (see [2]) and various types of generic distributions are examples of parabolic geometries. 
One basic principle in the study of parabolic geometries (and also of general Cartan geometries) is the passage from $G / P$, which in this context is referred to as the homogeneous model, and general (curved) Cartan geometries of type $(G, P)$. Sometimes, this passage is easy. For example, homogeneous vector bundles on $G / P$ are in bijective correspondence with representations of $P$. Given a representation $W$ of $P$ and a Cartan geometry $(p: \mathcal{G} \rightarrow M, \omega)$ of type $(G, P)$ one can form the associated bundle $W M:=\mathcal{G} \times{ }_{P} W$. The resulting bundles are called natural bundles on Cartan geometries of type $(G, P)$.

The Cartan connection $\omega$ can be used to identify such bundles with other types of natural bundles. The basic example is provided by the representation $\mathfrak{g} / \mathfrak{p}$. It is well known that the homogeneous bundle $G \times{ }_{P}(\mathfrak{g} / \mathfrak{p})$ is isomorphic to the tangent bundle $T(G / P)$. Likewise, via the Cartan connection $\omega$, one constructs an isomorphism $\mathcal{G} \times_{P}(\mathfrak{g} / \mathfrak{p}) \cong T M$. As in the homogeneous case, sections of natural bundles can be described via equivariant functions, i.e. $\Gamma\left(\mathcal{G} \times{ }_{P} W\right) \cong$ $C^{\infty}(\mathcal{G}, W)^{P}$, with the principal right action of $P$ on $\mathcal{G}$ replacing the right translations by elements of $P$ on $G$.

A $G$-equivariant vector bundle map between homogeneous vector bundles is given by a $P$ equivariant linear map between the inducing representations and hence extends to arbitrary Cartan geometries as well. This means, that $G$-equivariant maps between induced representations, which are tensorial (i.e. linear over $\left.C^{\infty}(G / P, \mathbb{R})\right)$ canonically extend to tensorial operators on all Cartan geometries. For more general intertwining operators between induced representations, the situation is much more complicated. In particular, the case of intertwining differential operators is related to questions on invariant differential operators on parabolic geometries, which is a very active area of research.

\subsection{The fundamental derivative}

Finding invariant differential operators is particularly difficult for bundles induced by irreducible representations of $P$. Allowing more general bundles, there is a basic set of invariant operators. The construction is easy to understand on the homogeneous model. Suppose that $W$ is any representation of $P$ and consider sections of the induced bundle $G \times{ }_{P} W$ as equivariant functions $f: G \rightarrow W$. For a vector field $\xi \in \mathfrak{X}(G)$, the derivative $\xi \cdot f$ will not be equivariant in general. This will be the case, however, if $\xi$ is invariant under right translations by elements of $P$. Now using the left trivialization of the tangent bundle $T G$, vector fields on $G$ correspond to smooth functions $X: G \rightarrow \mathfrak{g}$ via $\xi(g)=L_{X(g)}$. Such a vector field is invariant under right translations by elements of $P$ if and only if $X(g h)=\operatorname{Ad}\left(h^{-1}\right)(X(g))$ for all $g \in G$ and $h \in P$. Consequently, $P$-invariant vector fields on $G$ are in bijective correspondence with sections of the associated bundle $G \times_{P} \mathfrak{g}$.

This carries over to the curved case. Given a parabolic geometry $(p: \mathcal{G} \rightarrow M, \omega)$, one defines the adjoint tractor bundle $\mathcal{A} M:=\mathcal{G} \times{ }_{P} \mathfrak{g}$. Via the Cartan connection, smooth sections of $\mathcal{A} M$ are in bijective correspondence with $P$-invariant vector fields on $\mathcal{G}$. For the natural bundle $W M=\mathcal{G} \times{ }_{P} W$ corresponding to the $P$-representation $W$, one has $\Gamma(W M) \cong C^{\infty}(\mathcal{G}, W)^{P}$. Differentiating a $P$-equivariant function with respect to a $P$-invariant vector field, the result is $P$-equivariant again. Hence there is a bilinear natural differential operator

$$
D: \Gamma(\mathcal{A} M) \times \Gamma(\mathcal{W} M) \rightarrow \Gamma(\mathcal{W} M)
$$

To emphasize the analogy to a covariant derivative, this operator is usually denoted as $(s, \sigma) \mapsto$ $D_{s} \sigma$. The analogy is strengthened by the fact that $D$ is linear over $C^{\infty}(M, \mathbb{R})$ in the $\mathcal{A} M$-slot and satisfies a Leibniz rule in the other slot. This operator is called the fundamental derivative and was introduced (under the name "fundamental $D$-operator") in [5]. There it was also proved that this operator is compatible with any natural bundle map. Note that we can also view $D$ as 
an operator mapping $\Gamma(W M)$ to $\Gamma\left(\mathcal{A}^{*} M \otimes W M\right)$, and in this form the operator can be iterated without problems. Hence for a section $\sigma \in \Gamma(W M)$, we can form $D^{k} \sigma \in \Gamma\left(\otimes^{k} \mathcal{A}^{*} M \otimes W M\right)$.

We have to note one more important property of the fundamental derivative. The Lie subalgebra $\mathfrak{p} \subset \mathfrak{g}$ is a $P$-submodule. Hence for any parabolic geometry $(p: \mathcal{G} \rightarrow M, \omega)$ of type $(G, P)$, we obtain a subbundle $\mathcal{G} \times{ }_{P} \mathfrak{p} \subset \mathcal{G} \times{ }_{P} \mathfrak{g}=\mathcal{A} M$. For reasons that will become clear later on, we denote this subbundle by $\mathcal{A}^{0} M$. For any finite dimensional representation $W$ of $P$, we have the corresponding infinitesimal representation of $\mathfrak{p}$ on $W$. We can interpret this as an action, i.e. as a bilinear map $\mathfrak{p} \times W \rightarrow W$, and this map is $P$-equivariant. Passing to associated bundles, we obtain an induced bilinear bundle map $\mathcal{A}^{0} M \times W M \rightarrow W M$, which we write as $(s, \sigma) \mapsto s \bullet \sigma$.

Now sections of $\mathcal{A}^{0} M \subset \mathcal{A} M$ correspond to $P$-invariant vector fields on $\mathcal{G}$, which are mapped by $\omega$ to $\mathfrak{p} \subset \mathfrak{g}$. By definition of a Cartan connection, the value of such a vector field in each point coincides with a fundamental vector field, so sections of $\mathcal{A}^{0} M$ correspond to $P$-invariant vertical vector fields. But differentiating a $P$-equivariant function with respect to a fundamental vector field, one obtains the infinitesimal action of minus the generator of the fundamental field. This implies that $D_{s} \sigma=-s \bullet \sigma$ holds for all $s \in \Gamma\left(\mathcal{A}^{0} M\right)$ and $\sigma \in \Gamma(W M)$.

\subsection{A curved analog of the Casimir operator}

Given a (possibly infinite dimensional) representation $V$ of the Lie group $G$ and its Lie algebra $\mathfrak{g}$, one always has the Casimir operator mapping the representation to itself. Since $\mathfrak{g}$ is semisimple, its Killing form $B$ is non-degenerate, and hence provides an isomorphism $\mathfrak{g} \cong \mathfrak{g}^{*}$ of $G$-modules. Now consider a basis $\left\{\xi^{\ell}\right\}$ of $\mathfrak{g}$ and let $\left\{\xi_{\ell}\right\}$ be the dual basis with respect to $B$. Then the Casimir operator $\mathcal{C}: V \rightarrow V$ is defined by $\mathcal{C}(v):=\sum_{\ell} \xi^{\ell} \cdot \xi_{\ell} \cdot v$. It is easy to see that $\mathcal{C}$ is independent of the choice of the basis $\left\{\xi^{\ell}\right\}$ and $G$-equivariant.

In particular, we can use this idea on the space of smooth sections of a homogeneous bundle $G \times{ }_{P} W \rightarrow G / P$. In the picture of equivariant functions, we have seen in Section 2.1 that the $G$-action on $C^{\infty}(G, W)^{P}$ is given by $(g \cdot f)(\tilde{g}):=f\left(g^{-1} \tilde{g}\right)$. This immediately implies that the action can be differentiated to obtain an action of the Lie algebra $\mathfrak{g}$ of $G$. This action is explicitly given by $X \cdot f:=-R_{X} \cdot f$, where $R_{X} \in \mathfrak{X}(G)$ is the right invariant vector field generated by $X \in \mathfrak{g}$.

Given bases $\left\{\xi^{\ell}\right\}$ and $\left\{\xi_{\ell}\right\}$ of $\mathfrak{g}$ as above, we conclude that the Casimir operator on $C^{\infty}(G, W)^{P}$ is given by $\mathcal{C}(f)=\sum_{\ell} R_{\xi^{\ell}} \cdot R_{\xi_{\ell}} \cdot f$. In particular, $\mathcal{C}$ is a differential operator of order at most 2 . Note that from this formula, it is not evident that $\mathcal{C}$ is independent of the choice of basis.

We want to express the Casimir operator $\mathcal{C}$ acting on sections of an arbitrary homogeneous vector bundle via the fundamental derivative. The resulting formula then defines an operator on sections of the corresponding natural vector bundle on arbitrary curved parabolic geometries. We have noted above that the Killing form $B$ induces an isomorphism $\mathfrak{g} \rightarrow \mathfrak{g}^{*}$ of $G$-modules and hence of $P$-modules. Passing to associated bundles, we obtain a natural isomorphism $\mathcal{A} M \rightarrow \mathcal{A}^{*} M$ between the adjoint tractor bundle and its dual on any parabolic geometry. This in turn can be used to interpret the Killing form as a natural tensorial linear operator which maps sections of $\otimes^{2} \mathcal{A}^{*} M$ to smooth functions on $M$.

Proposition 1. Let $G$ be a semisimple Lie group, $P \subset G$ a parabolic subgroup, let $W$ be a representation of $P$, and consider the Casimir operator $\mathcal{C}: \Gamma\left(G \times{ }_{P} W\right) \rightarrow \Gamma\left(G \times{ }_{P} W\right)$. Then $\mathcal{C}$ can be written as the composition

$$
\Gamma(W(G / P)) \stackrel{D^{2}}{\longrightarrow} \Gamma\left(\otimes^{2} \mathcal{A}^{*}(G / P) \otimes W(G / P)\right) \stackrel{B \otimes \mathrm{id}_{W}}{\longrightarrow} \Gamma(W(G / P)) .
$$

Hence $(B \otimes \mathrm{id}) \circ D^{2}$ defines an extension of $\mathcal{C}$ to an invariant differential operator acting on sections of the natural bundle induced by $W$ for all parabolic geometries of type $(G, P)$. 
Proof. For any $X \in \mathfrak{g}$, the right invariant vector field $R_{X} \in \mathfrak{X}(G)$ is in particular invariant under right translations by elements of $P$. Hence it corresponds to a section $s_{X} \in \Gamma(\mathcal{A}(G / P))$, and the corresponding equivariant function $G \rightarrow \mathfrak{g}$ is given by $g \mapsto \operatorname{Ad}\left(g^{-1}\right) X$. For a basis $\left\{\xi^{\ell}\right\}$ of $\mathfrak{g}$ with dual basis $\xi_{\ell}$, we thus see that $\mathcal{C}$ can be written as $\mathcal{C}(\sigma)=\sum_{\ell} D_{s_{\xi \ell}} D_{s_{\xi_{\ell}}} \sigma$. Now naturality of the fundamental derivative easily implies that

$$
D^{2} \sigma\left(s_{1}, s_{2}\right)=D_{s_{1}} D_{s_{2}} \sigma-D_{D_{s_{1}} s_{2}} \sigma
$$

see $[5,3.6]$. For $X, Y \in \mathfrak{g}$, the function corresponding to $s_{Y}$ is $g \mapsto \operatorname{Ad}\left(g^{-1}\right) Y$, and differentiating this with respect to $R_{X}$, we immediately conclude that $D_{s_{X}} s_{Y}=s_{[Y, X]}$.

Since the value of the Casimir operator is independent of the choice of the basis, we may as well write $\mathcal{C}(\sigma)$ as

$$
\frac{1}{2} \sum_{\ell}\left(D_{s_{\xi \ell}} D_{s_{\xi_{\ell}}} \sigma+D_{s_{\xi_{\ell}}} D_{s_{\xi \ell}} \sigma\right)
$$

and since from above we know that $D_{s_{X}} s_{Y}$ is skew symmetric in $X$ and $Y$, this equals

$$
\frac{1}{2} \sum_{\ell}\left(D^{2} \sigma\left(s_{\xi^{\ell}}, s_{\xi_{\ell}}\right)+D^{2} \sigma\left(s_{\xi_{\ell}}, s_{\xi^{\ell}}\right)\right) .
$$

Invariance of the Killing form implies that $B\left(\operatorname{Ad}\left(g^{-1}\right) X, \operatorname{Ad}\left(g^{-1}\right) Y\right)=B(X, Y)$, which shows that for each $g \in P$, the elements $\left\{s_{\xi^{\ell}}(g P)\right\}$ and $\left\{s_{\xi_{\ell}}(g P)\right\}$ form dual bases for the fiber $\mathcal{A}_{g P}(G / P)$ with respect to the bundle map induced by the Killing form. Hence

$$
\sum_{\ell} D^{2} \sigma\left(s_{\xi^{\ell}}, s_{\xi_{\ell}}\right)=\sum_{\ell} D^{2} \sigma\left(s_{\xi_{\ell}}, s_{\xi^{\ell}}\right)=(B \otimes \mathrm{id})\left(D^{2} \sigma\right),
$$

and the result follows.

Definition. We will refer to the operator $\mathcal{C}:=(B \otimes$ id $) \circ D^{2}$ on general parabolic geometries as the curved Casimir operator.

\section{Basic properties of the curved Casimir operators}

\subsection{Naturality}

Having a formula for the Casimir in terms of the fundamental derivative, we can now study its basic properties not only on the homogeneous model, but at the same time on general curved parabolic geometries. The first consequence is that, like the fundamental derivative, the curved Casimir operator has very strong naturality properties.

Proposition 2. Let $W$ and $W^{\prime}$ be representations of $P, \Phi: W \rightarrow W^{\prime}$ a P-equivariant mapping, and let us also denote by $\Phi$ the induced natural bundle map. Denoting by $\mathcal{C}_{W}$ and $\mathcal{C}_{W^{\prime}}$ the curved Casimir operators on the natural bundles induced by $W$ and $W^{\prime}$, we get $\mathcal{C}_{W^{\prime}}(\Phi(\sigma))=\Phi\left(\mathcal{C}_{W}(\sigma)\right)$ for any parabolic geometry $(p: \mathcal{G} \rightarrow M, \omega)$ of type $(G, P)$ and any $\sigma \in \Gamma(W M)$.

In particular, the curved Casimir preserves sections of natural subbundles and restricts to the curved Casimir of the subbundle on such sections. Likewise, the induced operator on sections of a natural quotient bundle coincides with the curved Casimir on that bundle.

Proof. By Proposition 3.1 of [5], the fundamental derivative has the property that $D_{s}(\Phi(\sigma))=$ $\Phi\left(D_{s} \sigma\right)$ for any $(p: \mathcal{G} \rightarrow M, \omega), s \in \Gamma(\mathcal{A} M)$ and $\sigma \in \Gamma(W M)$. Therefore, naturality follows directly from the definition of $\mathcal{C}$.

The second part of the claim is just the specialization to the inclusion of a subrepresentation respectively the projection to a quotient. 


\subsection{Adapted local frames}

To derive further properties of the curved Casimir, we will use a formula in terms of appropriately chosen local dual frames for the adjoint tractor bundle. This needs a bit more background about parabolic subalgebras.

A basic structural result on parabolic subgroups $P \subset G$ is the existence of a Langlands decomposition, which is usually written as $P=M A N$. Here $M \subset P$ is a semisimple Lie subgroup and $A, N \subset P$ are normal subgroups which are Abelian and nilpotent, respectively. In the literature on parabolic geometries, one usually considers the reductive subgroup $M A \subset P$, which is commonly denoted by $G_{0}$, while the nilpotent normal subgroup $N$ is usually denoted by $P_{+}$. On the level of Lie algebras, one has $\mathfrak{p}_{+} \subset \mathfrak{p} \subset \mathfrak{g}$ and $\mathfrak{p} / \mathfrak{p}_{+}=\mathfrak{g}_{0}$.

There is a finer decomposition however, see [18]. It turns out that there is a grading $\mathfrak{g}=$ $\mathfrak{g}_{-k} \oplus \cdots \oplus \mathfrak{g}_{k}$ of $\mathfrak{g}$ such that $\mathfrak{p}=\mathfrak{g}_{0} \oplus \cdots \oplus \mathfrak{g}_{k}$ and $\mathfrak{p}_{+}=\mathfrak{g}_{1} \oplus \cdots \oplus \mathfrak{g}_{k}$. This grading is only an auxiliary tool for parabolic geometries, since it is not $\mathfrak{p}$-invariant. The corresponding filtration

$$
\mathfrak{g}=\mathfrak{g}^{-k} \supset \mathfrak{g}^{-k+1} \supset \cdots \supset \mathfrak{g}^{k} \supset \mathfrak{g}^{k+1}=\{0\}
$$

defined by $\mathfrak{g}^{i}:=\mathfrak{g}_{i} \oplus \cdots \oplus \mathfrak{g}_{k}$ is $P$-invariant. In this notation $\mathfrak{p}=\mathfrak{g}^{0}$ and $\mathfrak{p}_{+}=\mathfrak{g}^{1}$, so the facts that $\mathfrak{p} \subset \mathfrak{g}$ is a subalgebra and that $\mathfrak{p}_{+} \subset \mathfrak{p}$ is a nilpotent ideal immediately follow from that fact that the filtration is compatible with the Lie bracket.

For any parabolic geometry $(p: \mathcal{G} \rightarrow M, \omega)$ of type $(G, P)$, the $P$-invariant filtration of $\mathfrak{g}$ gives rise to a filtration $\mathcal{A} M=\mathcal{A}^{-k} M \supset \cdots \supset \mathcal{A}^{k} M$ of the adjoint tractor bundle by smooth subbundles. In particular, $\mathcal{A}^{0} M=\mathcal{G} \times_{P} \mathfrak{p}$, which justifies the notation introduced before. If $U \subset M$ is an open subset over which $\mathcal{G}$ is trivial, then all the bundles $\mathcal{A}^{i} M$ are trivial over $U$. Over such a subset, we can therefore choose local frames of these subbundles.

From the facts that the grading and the induced filtration of $\mathfrak{g}$ are compatible with the Lie bracket, one can easily deduce the relation of the Killing form $B$ to these data, see again [18]. For $X \in \mathfrak{g}_{i}$ and $Y \in \mathfrak{g}_{j}, \operatorname{ad}_{X} \circ \operatorname{ad}_{Y}$ maps $\mathfrak{g}_{\ell}$ to $\mathfrak{g}_{i+j+\ell}$. Hence this composition is tracefree and $B$ vanishes on $\mathfrak{g}_{i} \times \mathfrak{g}_{j}$ unless $j=-i$. Consequently, $B$ is non-degenerate on $\mathfrak{g}_{0}$ and induces dualities of $G_{0}$-modules between $\mathfrak{g}_{i}$ and $\mathfrak{g}_{-i}$ for all $i=1, \ldots, k$. Likewise, for $i=-k+1, \ldots, k, \mathfrak{g}^{i}$ is the annihilator with respect to $B$ of $\mathfrak{g}^{-i+1}$, and one obtains dualities of $P$-modules between $\mathfrak{g}^{i}$ and $\mathfrak{g} / \mathfrak{g}^{-i+1}$. Translated to associated bundles, this implies that $B$ vanishes on $\mathcal{A}^{-i+1} M \times \mathcal{A}^{i} M$ and induces a non-degenerate bilinear form on $\left(\mathcal{A} M / \mathcal{A}^{-i+1} M\right) \times \mathcal{A}^{i} M$. Since $\mathcal{A}^{0} M / \mathcal{A}^{1} M \cong$ $\mathcal{G} \times{ }_{P} \mathfrak{g}_{0}$, the Killing form induces a non-degenerate bilinear form $B$ on this bundle.

Definition. Consider the adjoint tractor bundle $\mathcal{A} M$ for a parabolic geometry of type $(G, P)$, and consider the grading and the filtration of $\mathfrak{g}$ induced by the parabolic subalgebra $\mathfrak{p} \subset \mathfrak{g}$. Then an adapted local frame for $\mathcal{A} M$ is a local frame of the form

$$
\left\{X_{i}, A_{r}, Z^{i}: i=1, \ldots, \operatorname{dim}\left(\mathfrak{p}_{+}\right), r=1, \ldots, \operatorname{dim}\left(\mathfrak{g}_{0}\right)\right\},
$$

such that

- $Z^{i} \in \Gamma\left(\mathcal{A}^{1} M\right)$ for all $i$ and $A_{r} \in \Gamma\left(\mathcal{A}^{0} M\right)$ for all $r$ (which implies $B\left(Z^{i}, Z^{j}\right)=0$ and $B\left(Z^{i}, A_{r}\right)=0$ for all $i, j, r$ by the properties of $B$ ).

- $B\left(X_{i}, X_{j}\right)=B\left(X_{i}, A_{r}\right)=0$, and $B\left(X_{i}, Z^{j}\right)=\delta_{i}^{j}$ for all $i, j, r$.

Lemma 1. Adapted frames always exist locally. Moreover, if $\left\{X_{i}, A_{r}, Z^{i}\right\}$ is an adapted local frame, then the dual frame with respect to $B$ has the form $\left\{Z^{i}, A^{r}, X_{i}\right\}$ for certain sections $A^{r} \in \Gamma\left(\mathcal{A}^{0} M\right)$. The sections $A_{r}$ and $A^{r}$ project to local frames of $\mathcal{A}^{0} M / \mathcal{A}^{1} M$, which are dual with respect to $B$. 
Proof. Consider an open subset $U \subset M$ over which $\mathcal{G}$ is trivial. Then over $U$, each of the bundles $\mathcal{A}^{i} M$ is trivial and hence admits a local frame. We have noted above that $B$ induces a duality between the bundles $\mathcal{A}^{1}$ and $\mathcal{A} / \mathcal{A}^{0}$ and a non-degenerate bilinear form on $\mathcal{A}^{0} / \mathcal{A}^{1}$. Hence we can choose dual local frames $\left\{Z^{i}\right\}$ of $\mathcal{A}^{1}$ and $\left\{\underline{X}_{i}\right\}$ of $\mathcal{A} / \mathcal{A}^{0}$ as well as an arbitrary local frame $\left\{\underline{A}_{r}\right\}$ of $\mathcal{A}^{0} / \mathcal{A}^{1}$. Choosing arbitrary representatives $\tilde{X}_{i} \in \Gamma(\mathcal{A})$ and $\tilde{A}_{r} \in \Gamma\left(\mathcal{A}^{0}\right)$ for the $\underline{X}_{i}$ and the $\underline{A}_{r}$, the set $\left\{\tilde{X}_{i}, \tilde{A}_{r}, Z^{i}\right\}$ forms a local frame for $\mathcal{A}$.

Given such a choice, we put $X_{i}:=\tilde{X}_{i}-\sum_{j} \frac{1}{2} B\left(\tilde{X}_{i}, \tilde{X}_{j}\right) Z^{j}$. Then $B\left(X_{i}, X_{j}\right)=0$ and $B\left(X_{i}, Z^{j}\right)=\delta_{i}^{j}$ hold by construction. Next, defining $A_{r}:=\tilde{A}_{r}-\sum_{i} B\left(X_{i}, \tilde{A}_{r}\right) Z^{i}$ we also get $B\left(X_{i}, A_{r}\right)=0$, so $\left\{X_{i}, A_{r}, Z^{i}\right\}$ is an adapted local frame.

The form $B$ restricts to zero on $\mathcal{A}^{0} M \times \mathcal{A}^{1} M$, so $B\left(Z^{i}, Z^{j}\right)=0$ and $B\left(Z^{i}, A_{r}\right)=0$ for $i, j$, and $r$. This shows that the dual frame to $\left\{X_{i}, A_{r}, Z^{i}\right\}$ must have the $Z^{i}$ as its first elements and the $X_{i}$ as its last elements. Denoting the remaining elements by $A^{r}$, we by definition have $B\left(A^{r}, Z^{i}\right)=0$ for all $r$ and $i$. Since $\mathcal{A}^{0} M$ is the annihilator of $\mathcal{A}^{1} M$ with respect to $B$, this shows that $A^{r} \in \Gamma\left(\mathcal{A}^{0} M\right)$ for all $r$. The last claim then is obvious.

\subsection{A formula for the curved Casimir}

The key step in our analysis of the curved Casimir operator is that it admits a simple local formula in terms of an adapted frame. To formulate this, we need a final ingredient. The Lie bracket on $\mathfrak{g}$ defines a $P$-equivariant bilinear map [, ] $: \mathfrak{g} \times \mathfrak{g} \rightarrow \mathfrak{g}$. Passing to induced bundles, we obtain a skew symmetric bilinear bundle map $\{\}:, \mathcal{A} M \times \mathcal{A} M \rightarrow \mathcal{A} M$. By construction, this makes each fiber $\mathcal{A}_{x} M$ into a filtered Lie algebra isomorphic to $\left(\mathfrak{g},\left\{\mathfrak{g}^{i}\right\}\right)$.

Proposition 3. Let $(p: \mathcal{G} \rightarrow M, \omega)$ be a parabolic geometry of type $(G, P)$, and for a representation $W$ of $P$ consider the natural bundle $W M=\mathcal{G} \times{ }_{P} W$. Let $\mathcal{C}: \Gamma(W M) \rightarrow \Gamma(W M)$ be the curved Casimir operator. Let $U \subset M$ be an open subset and $\left\{X_{i}, A_{r}, Z^{i}\right\}$ be an adapted frame for the adjoint tractor bundle $\mathcal{A} M$ over $U$ with dual frame $\left\{Z^{i}, A^{r}, X_{i}\right\}$. Then for a section $\sigma \in \Gamma(W M)$ we get

$$
\left.\mathcal{C}(\sigma)\right|_{U}=-2 \sum_{i} Z^{i} \bullet D_{X_{i}} \sigma-\sum_{i}\left\{Z^{i}, X_{i}\right\} \bullet \sigma+\sum_{r} A^{r} \bullet A_{r} \bullet \sigma .
$$

Proof. We can use the adapted frame and its dual to evaluate $\mathcal{C}(\sigma)$ as

$$
\sum_{i}\left(D^{2} \sigma\left(X_{i}, Z^{i}\right)+D^{2} \sigma\left(Z^{i}, X_{i}\right)\right)+\sum_{r} D^{2} \sigma\left(A_{r}, A^{r}\right)
$$

and then use equation (1) from Section 2.4 to evaluate the individual summands. All $Z^{i}, A_{r}$, and $A^{r}$ are sections of $\Gamma\left(\mathcal{A}^{0} M\right)$, so if $s$ is any of these sections then $D_{s}$ acts as $-s \bullet$. Naturality of $D$ further implies that for any $s \in \Gamma(\mathcal{A} M)$, the operator $D_{s}$ on $\Gamma(\mathcal{A} M)$ is filtration preserving. Finally, the map $\bullet: \mathcal{A}^{0} M \times \mathcal{A} M \rightarrow \mathcal{A} M$ coincides with $\{$,$\} and \left\{Z^{i}, X_{i}\right\} \in \Gamma\left(\mathcal{A}^{0}\right)$ for all $i$. Using these facts, the first sum in (2) gives

$$
\sum_{i}\left(-D_{X_{i}}\left(Z_{i} \bullet \sigma\right)+\left(D_{X_{i}} Z^{i}\right) \bullet \sigma-Z_{i} \bullet D_{X_{i}} \sigma-\left\{Z^{i}, X_{i}\right\} \bullet \sigma\right)
$$

and by naturality of $D_{X_{i}}$ the first two terms add up to $-Z_{i} \bullet D_{X_{i}} \sigma$. On the other hand, for the last sum in (2), we obtain

$$
A_{r} \bullet A^{r} \bullet \sigma-\left\{A_{r}, A^{r}\right\} \bullet \sigma=A^{r} \bullet A_{r} \bullet \sigma,
$$

and the result follows. 


\subsection{More basic properties of the curved Casimir}

One of the main results will be the action of the curved Casimir operator on bundles induced by irreducible representations. Recall that $P$ contains the nilpotent normal subgroup $P_{+}$, and $P / P_{+} \cong G_{0}$. It turns out that $P_{+}$acts trivially on any irreducible representations of $P$, so any such representation comes from an irreducible representation of the reductive group $G_{0}$. To formulate the result, we will need some information on weights.

If $\mathfrak{g}$ is a complex semisimple Lie algebra, then we can choose a Cartan subalgebra $\mathfrak{h} \subset \mathfrak{g}$ and positive roots $\Delta^{+}$in such a way that $\mathfrak{p} \subset \mathfrak{g}$ is a standard parabolic subalgebra, i.e. contains $\mathfrak{h}$ and all positive root spaces. Then $\mathfrak{h} \subset \mathfrak{g}_{0}$ and $\mathfrak{h}$ is also a Cartan subalgebra for the reductive Lie algebra $\mathfrak{g}_{0}$, i.e. it is the direct sum of the center $\mathfrak{z}\left(\mathfrak{g}_{0}\right)$ and a Cartan subalgebra of the semisimple part of $\mathfrak{g}_{0}$. Hence weights for both $\mathfrak{g}$ and $\mathfrak{g}_{0}$ are linear functionals on $\mathfrak{h}$. Our results are easier to formulate in terms of lowest weights than in terms of highest weights. By $\rho$, we will denote the lowest form of $\mathfrak{g}$, i.e. half the sum of all positive roots, or, equivalently, the sum of all fundamental weights.

Suppose now that $\mathfrak{g}$ is a real semisimple Lie algebra. Then the grading $\mathfrak{g}=\oplus_{i=-k}^{k} \mathfrak{g}_{i}$ induces a grading of the complexification $\mathfrak{g}_{\mathbb{C}}$ and hence $\mathfrak{p}_{\mathbb{C}} \subset \mathfrak{g}_{\mathbb{C}}$ is a parabolic subalgebra. Now one can find a subalgebra $\mathfrak{h} \subset \mathfrak{g}$ such that $\mathfrak{h}_{\mathbb{C}} \subset \mathfrak{g}_{\mathbb{C}}$ is a Cartan subalgebra for which $\mathfrak{p}_{\mathbb{C}}$ is a standard parabolic. Then we can interpret weights for $\mathfrak{g}_{\mathbb{C}}$ as well as for $\left(\mathfrak{g}_{0}\right)_{\mathbb{C}}$ as linear functionals on $\mathfrak{h}$. If $W$ is a complex irreducible representation of $\mathfrak{g}_{0}$, then it extends to $\left(\mathfrak{g}_{0}\right)_{\mathbb{C}}$, and thus has a lowest weight. If $W$ is irreducible without an invariant complex structure, then the complexification $W_{\mathbb{C}}$ is a complex irreducible representation of $\mathfrak{g}_{0}$, and we refer to the lowest weight of $W_{\mathbb{C}}$ also as the lowest weight of $W$.

Theorem 1. (1) On any natural vector bundle, the curved Casimir operator is a differential operator of order at most one.

(2) Let $W$ be an irreducible representation of $P$. The the curved Casimir operator acts by a scalar on $\Gamma(W M)$ for any $(p: \mathcal{G} \rightarrow M, \omega)$. If $-\nu$ is the lowest weight of $W$, then this scalar is explicitly given by

$$
\|\nu+\rho\|^{2}-\|\rho\|^{2}=\langle\nu, \nu\rangle+2\langle\nu, \rho\rangle,
$$

where the norms and inner products are induced by the Killing form.

Proof. (1) is obvious from Proposition 3, since in the formula for $\left.\mathcal{C}(\sigma)\right|_{U}$, the term $D_{X_{i}} \sigma$ is first order in $\sigma$, while all other ingredients are tensorial.

(2) If $W$ is irreducible, then we know that $P_{+}$and hence $\mathfrak{p}_{+}$acts trivially on $W$. Then the formula from Proposition 3 reduces to

$$
\left.\mathcal{C}(\sigma)\right|_{U}=-\sum_{i}\left\{Z^{i}, X_{i}\right\} \bullet \sigma+\sum_{r} A^{r} \bullet A_{r} \bullet \sigma
$$

so in particular, $\mathcal{C}$ is tensorial and acts only via the values of $\sigma$. By construction, this action is induced by the map $W \rightarrow W$ given by

$$
w \mapsto-\sum_{i}\left[Z^{i}, X_{i}\right] \cdot w+\sum_{r} A^{r} \cdot A_{r} \cdot w,
$$

where now $\left\{Z^{i}\right\}$ and $\left\{X_{i}\right\}$ are bases of the $\mathfrak{g}_{0}$-modules $\mathfrak{p}_{+}$and $\mathfrak{g}_{-}=\mathfrak{g}_{-k} \oplus \cdots \oplus \mathfrak{g}_{-1} \cong\left(\mathfrak{p}_{+}\right)^{*}$ which are dual with respect to $B$, while $\left\{A_{r}\right\}$ and $\left\{A^{r}\right\}$ are dual bases of $\mathfrak{g}_{0}$ with respect to $B$. In this formula, we only use dual bases of dual $\mathfrak{g}_{0}$-modules as well as the brackets $[]:, \mathfrak{g}_{i} \times \mathfrak{g}_{-i} \rightarrow \mathfrak{g}_{0}$ and the action of $\mathfrak{g}_{0}$ on $W$, which all are $\mathfrak{g}_{0}$-equivariant. Thus $(3)$ is a homomorphism of $\mathfrak{g}_{0}$-modules, and hence a scalar multiple of the identity by irreducibility.

To compute this scalar, we may, if necessary, first complexify $W$ and then complexify $\mathfrak{g}$. Hence we may without loss of generality assume that $\mathfrak{g}$ is complex and $W$ is a complex representation 
of $\mathfrak{g}$. Now we choose a Cartan subalgebra $\mathfrak{h} \subset \mathfrak{g}$ such that $\mathfrak{p} \subset \mathfrak{g}$ is a standard parabolic subalgebra. Then $\mathfrak{h}$ and all positive root spaces are contained in $\mathfrak{p}=\mathfrak{g}_{0} \oplus \mathfrak{p}_{+}$. For $\alpha \in \Delta^{+}$the root space $\mathfrak{g}_{\alpha}$ is thus either contained in $\mathfrak{g}_{0}$ or in $\mathfrak{p}_{+}$and in the first case $\mathfrak{g}_{-\alpha} \in \mathfrak{g}_{0}$ while in the second case $\mathfrak{g}_{-\alpha} \in \mathfrak{g}_{-}$. Accordingly, we decompose $\Delta^{+}$into the disjoint union $\Delta^{+}\left(\mathfrak{g}_{0}\right) \sqcup \Delta^{+}\left(\mathfrak{p}_{+}\right)$.

For each root $\alpha \in \Delta^{+}$choose elements $E_{\alpha} \in \mathfrak{g}_{\alpha}$ and $F_{\alpha} \in \mathfrak{g}_{-\alpha}$ such that $B\left(E_{\alpha}, F_{\alpha}\right)=1$. Then $\left[E_{\alpha}, F_{\alpha}\right]=H_{\alpha} \in H$, the element dual to $\alpha$ with respect to $B$. Let $\left\{H_{\ell}: \ell=1, \ldots, r\right\}$ be an orthonormal basis of $\mathfrak{h}$ with respect to $B$ and put

$$
\begin{aligned}
& \left\{X_{i}\right\}:=\left\{F_{\alpha}: \alpha \in \Delta^{+}\left(\mathfrak{p}_{+}\right)\right\} \quad\left\{Z^{i}\right\}:=\left\{E_{\alpha}: \alpha \in \Delta^{+}\left(\mathfrak{p}_{+}\right)\right\} \\
& \left\{A_{r}\right\}:=\left\{E_{\alpha}, F_{\alpha}: \alpha \in \Delta^{+}\left(\mathfrak{g}_{0}\right)\right\} \cup\left\{H_{\ell}: \ell=1, \ldots, r\right\} .
\end{aligned}
$$

Then these are bases as required, and for the dual basis to $\left\{A_{r}\right\}$ we evidently get

$$
\left\{A^{r}\right\}:=\left\{F_{\alpha}, E_{\alpha}: \alpha \in \Delta^{+}\left(\mathfrak{g}_{0}\right)\right\} \cup\left\{H_{\ell}: \ell=1, \ldots, r\right\} .
$$

Using these bases, we can now evaluate (3) as

$$
-\sum_{\alpha \in \Delta^{+}\left(\mathfrak{p}_{+}\right)}\left[E_{\alpha}, F_{\alpha}\right] \cdot w+\sum_{\alpha \in \Delta^{+}\left(\mathfrak{g}_{0}\right)}\left(E_{\alpha} \cdot F_{\alpha}+F_{\alpha} \cdot E_{\alpha}\right) \cdot w+\sum_{\ell=1}^{r} H_{\ell} \cdot H_{\ell} \cdot w .
$$

Each summand in the middle sum can be rewritten as $\left(2 E_{\alpha} \cdot F_{\alpha}-\left[E_{\alpha}, F_{\alpha}\right]\right) \cdot w$. Now if $w \in W$ is a lowest weight vector, then each $F_{\alpha}$ acts trivially on $w$, and we are left with

$$
-\sum_{\alpha \in \Delta^{+}} H_{\alpha} \cdot w+\sum_{\ell=1}^{r} H_{\ell} \cdot H_{\ell} \cdot w .
$$

Writing the weight of $w$ as $-\nu$, each summand in the first sum produces $\langle\alpha, \nu\rangle$, and summing up we obtain $2\langle\rho, \nu\rangle$. Since $\left\{H_{\ell}\right\}$ is an orthonormal basis, the second sum simply contributes $\langle-\nu,-\nu\rangle=\|\nu\|^{2}$.

\subsection{An application}

Under weak assumptions, finite dimensional representations of $P$ admit finite composition series by completely reducible representations. Correspondingly, any natural bundle has many irreducible subquotients. Using the naturality properties of the curved Casimir operators together with the knowledge of the action on sections of irreducible bundles, we can construct so-called splitting operators. These are invariant differential operator mapping sections of some subquotient injectively to sections of the original bundle. We just describe the general procedure here and do not go into specific examples.

Given a representation $W$ of $P$, the composition series is obtained as follows. For an integer $N$ to be specified later, we put $W^{N}:=W^{\mathfrak{p}_{+}}$, the set of $\mathfrak{p}_{+}$-invariant elements of $W$. Then $W^{N} \subset W$ is easily seen to be $P$-invariant, and by construction, $\mathfrak{p}_{+}$acts trivially on $W^{N}$. Next, one defines $W^{N-1}:=\left\{w \in W: Z \cdot w \in W^{N} \quad \forall Z \in \mathfrak{p}_{+}\right\}$. Again, this is a $P$-invariant subspace of $W$, it contains $W^{N}$, and $\mathfrak{p}_{+}$acts trivially on $W^{N-1} / W^{N}$. Then one continues inductively and arranges $N$ in such a way that one obtains a filtration of the form $W=W^{0} \supset W^{1} \supset \cdots \supset W^{N}$. If the center of $\mathfrak{g}_{0}$ acts diagonalizably on the quotients $W^{i} / W^{i+1}$, then all these quotients are completely reducible. By Theorem 1 the curved Casimir then acts on each irreducible component of this quotient by a scalar that can be computed from the highest weight.

Theorem 2. Let $W$ be a representation of $P$ endowed with a $P$-invariant filtration $W=W^{0} \supset$ $W^{1} \supset \cdots \supset W^{N} \supset W^{N+1}=\{0\}$ such that each of the subquotients $W^{j} / W^{j+1}$ is completely reducible. Fix a number $i \in\{0, \ldots, N\}$ and an irreducible component $W^{\prime} \subset W^{i} / W^{i+1}$, and 
let $\mu_{0}$ be the eigenvalue of the curved Casimir operator on the natural bundle induced by $W^{\prime}$. Further, for each $j>i$ let $\mu_{1}^{j}, \ldots, \mu_{n_{j}}^{j}$ be the different eigenvalues of the curved Casimir operator on the bundles induced by the irreducible components of $W^{j} / W^{j+1}$.

Let $(p: \mathcal{G} \rightarrow M, \omega)$ be a parabolic geometry of type $(G, P)$. Then the natural operator $L:=\prod_{j=i+1}^{N} \prod_{k=1}^{n_{j}}\left(\mathcal{C}-\mu_{k}^{j}\right)$ on $\Gamma(W M)$ descends to an operator $\Gamma\left(W^{\prime} M\right) \rightarrow \Gamma\left(W^{i} M\right)$. Denoting by $\pi$ the natural tensorial projection $\Gamma\left(W^{i} M\right) \rightarrow \Gamma\left(W^{i} M / W^{i+1} M\right)$, we get $\pi(L(\sigma))=$ $\left(\prod_{j=i+1}^{n} \prod_{k=1}^{n_{j}}\left(\mu^{0}-\mu_{k}^{j}\right)\right) \sigma$. In particular, if all the $\mu_{k}^{j}$ are different from $\mu_{0}$, then $L$ defines a natural splitting operator.

Proof. For $j=i+1, \ldots, N$ put $L_{j}:=\prod_{k=1}^{n_{j}}\left(\mathcal{C}-\mu_{k}^{j}\right)$. Then $L=L_{i+1} \circ \cdots \circ L_{N}$, and all the operators $L_{j}$ commute with each other. By Proposition 2, $\mathcal{C}$ preserves sections of each of the subbundles $W^{j} M$, so in particular, we can initially interpret $L$ and the $L_{j}$ as operators $\Gamma\left(W^{k} M\right) \rightarrow \Gamma\left(W^{k} M\right)$ for each $k$.

Now take $j \in\{i+1, \ldots, N\}$ and let $\tilde{W} \subset W^{j} / W^{j+1}$ be an irreducible component. Then we obtain a natural subbundle $\tilde{W} M \subset W^{j} M / W^{j+1} M$, and we denote by $\pi$ the natural projection $W^{j} M \rightarrow W^{j} M / W^{j+1} M$, as well as the induced tensorial operator on sections. For $\sigma \in \Gamma(\tilde{W} M)$ we can choose $\hat{\sigma} \in \Gamma\left(W^{j} M\right)$ such that $\pi(\hat{\sigma})=\sigma$. Now $\pi^{-1}(\tilde{W} M) \subset W^{j} M$ is a natural subbundle, so by Proposition $2, \pi(\mathcal{C}(\hat{\sigma})) \in \Gamma(\tilde{W} M)$ and the image coincides with the action of the curved Casimir of the bundle $\tilde{W} M$ on $\sigma$. Hence $\pi(\mathcal{C}(\hat{\sigma}))=\mu_{k}^{j} \sigma$ for some $k=1, \ldots, n_{j}$. But this implies that $\left(\mathcal{C}-\mu_{k}^{j}\right)(\hat{\sigma}) \in \Gamma\left(W^{j+1} M\right)$, and since the composition defining $L_{j}$ can be written in arbitrary order, we see that $L_{j}(\hat{\sigma}) \in \Gamma\left(W^{j+1} M\right)$. Since this holds for any irreducible component, we see that $L_{j}$ maps $\Gamma\left(W^{j} M\right)$ to $\Gamma\left(W^{j+1} M\right)$.

Applied to $j=N$, this says that $L_{N}$ vanishes on $\Gamma\left(W^{N} M\right)$. Thus $L_{N} \circ L_{N-1}$ vanishes on $\Gamma\left(W^{N-1} M\right)$, and inductively, we conclude that $L$ vanishes on $\Gamma\left(W^{i+1} M\right)$. Hence it descends to an operator $\Gamma\left(W^{i} M / W^{i+1} M\right) \rightarrow \Gamma\left(W^{i} M\right)$, which we may restrict to sections of the natural subbundle $W^{\prime} M$, so $L$ descends as required. In terms of the natural projection $\pi: \Gamma\left(W^{i} M\right) \rightarrow$ $\Gamma\left(W^{i} M / W^{i+1} M\right)$ this means that for a section $\hat{\sigma} \in \Gamma\left(W^{i} M\right)$, the value of $L(\hat{\sigma})$ depends only on $\pi(\hat{\sigma})$. Supposing that $\pi(\hat{\sigma}) \in \Gamma\left(W^{\prime} M\right)$, Proposition 2 and Theorem 1 imply that we have $\pi(L(\hat{\sigma}))=\left(\prod_{j=i+1}^{N} \prod_{k=1}^{n_{j}}\left(\mathcal{C}-\mu_{k}^{j}\right)\right) \pi(\hat{\sigma})$, where now $\mathcal{C}$ is the curved Casimir on $\Gamma\left(W^{\prime} M\right)$. Since this acts by multiplication by $\mu_{0}$, the result follows.

Let us remark that the number of factors in the definition of $L$ may sometimes be reduced by passing to the $P$-submodule of $W$ generated by $W^{\prime}$.

\section{Tractor bundles and the BGG machinery}

In this last section, we analyze the action of the curved Casimir operator on sections of tractor bundles and on differential forms with values in a tractor bundle. In particular, this will lead to a nice interpretation of the resolutions of irreducible representations of $\mathfrak{g}$ by (smooth vectors in) principal series representations obtained via BGG sequences.

\subsection{Tractor bundles}

As before, let $P \subset G$ be a parabolic subgroup in a semisimple Lie group. Then any representation $V$ of $G$ can be restricted to obtain a representation of $P$. The corresponding natural bundles for parabolic geometries of type $(G, P)$ are called tractor bundles and play an important role in the theory of parabolic geometries. The map $G \times V \rightarrow G / P \times V$ defined by $(g, v) \mapsto(g P, g \cdot v)$ induces an isomorphism of homogeneous vector bundles over the homogeneous model $G / P$, and hence $\Gamma\left(G \times{ }_{P} V\right) \cong C^{\infty}(G / P, \mathbb{R}) \otimes V$ as a representation of $G$. However, for a parabolic geometry $(p: \mathcal{G} \rightarrow M, \omega)$, the natural bundle $V M=\mathcal{G} \times{ }_{P} V$ is in general not trivial. 
Via the trivialization constructed above, the bundle $G \times_{P} V$ inherits a canonical $G$-invariant linear connection. Such a natural connection on a tractor bundle also exists for arbitrary parabolic geometries, which is the main reason for the importance of these bundles. The general theory of these tractor connections is developed in [5]. For our purposes, the simplest description of the tractor connection is the following. Since $V$ is a representation of $G$, the infinitesimal action of $\mathfrak{p}$ extends to $\mathfrak{g}$. Hence the bundle map $\bullet$ introduced in Section 2.3 extends to a bundle map $\mathcal{A} M \times V M \rightarrow V M$, which has the properties of a Lie algebra action.

Now consider the operator $\Gamma(\mathcal{A} M) \times \Gamma(V M) \rightarrow \Gamma(V M)$ defined by $(s, \sigma) \mapsto D_{s} \sigma+s \bullet \sigma$. This is linear over $C^{\infty}(M, \mathbb{R})$ in the $\mathcal{A} M$-slot and satisfies a Leibniz rule in the $V M$-slot. From Section 2.3 we know that for $s \in \Gamma\left(\mathcal{A}^{0} M\right)$, we get $D_{s} \sigma=-s \bullet \sigma$, so our bundle map descends to $\Gamma\left(\mathcal{A} M / \mathcal{A}^{0} M\right)$ in the first argument. By construction, $\mathcal{A} M / \mathcal{A}^{0} M=\mathcal{G} \times_{P} \mathfrak{g} / \mathfrak{p} \cong T M$. Hence our bundle map descends to a bundle map $\mathfrak{X}(M) \times \Gamma(V M) \rightarrow \Gamma(V M)$, which is linear over smooth functions in the vector field and satisfies a Leibniz rule in the other slot. Thus it defines a natural linear connection $\nabla^{V}$ on $V M$.

We have noted already that the natural bundle corresponding to $\mathfrak{g} / \mathfrak{p}$ is the tangent bundle. Moreover, from Section 3.2 we know that $(\mathfrak{g} / \mathfrak{p})^{*} \cong \mathfrak{p}_{+}$as a $P$-module, so the natural bundle corresponding to $\mathfrak{p}_{+}$is the cotangent bundle. In particular, for a parabolic geometry on $M$, the cotangent bundle $T^{*} M$ naturally is a bundle of nilpotent filtered Lie algebras modeled on $\mathfrak{p}_{+}$. Moreover, the cotangent bundle $T^{*} M$ can be naturally identified with the subbundle $\mathcal{A}^{1} M$ of the adjoint tractor bundle. Given a representation $W$ of $P$, the bundles of differential forms with values in the natural bundle $W M$ correspond to the representations $\Lambda_{\mathfrak{p}_{+}}^{k} W$.

Now this gives rise to another natural operation. Via the infinitesimal action, $\mathfrak{p}_{+}$acts on any representation $W$ of $P$. The corresponding bundle map is simply the restriction $\bullet: \mathcal{A}^{1} M \times$ $W M \rightarrow W M$ of the bundle map introduced in Section 2.3. Otherwise put, $W M$ is a bundle of modules over the bundle $T^{*} M$ of Lie algebras. Now the Lie algebra homology differential defines a natural map $\Lambda^{k} \mathfrak{p}_{+} \otimes W \rightarrow \Lambda^{k-1} \mathfrak{p}_{+} \otimes W$. In the literature on parabolic geometries, this operation is mainly considered in the case of a representation of $\mathfrak{g}$ and it is called the Kostant codifferential and denoted by $\partial^{*}$, since it was introduced in [14] as the adjoint of a Lie algebra cohomology differential. Explicitly, the corresponding bundle map $\partial^{*}: \Lambda^{k} T^{*} M \otimes W M \rightarrow \Lambda^{k-1} T^{*} M \otimes W M$ is on decomposable elements given by

$$
\begin{aligned}
\partial^{*}\left(\varphi_{1} \wedge \cdots \wedge \varphi_{k} \otimes \sigma\right):= & \sum_{i=1}^{k}(-1)^{i} \varphi_{1} \wedge \cdots \wedge \widehat{\varphi}_{i} \wedge \cdots \wedge \varphi_{k} \otimes \varphi_{i} \bullet \sigma \\
& +\sum_{i<j}(-1)^{i+j}\left\{\varphi_{i}, \varphi_{j}\right\} \wedge \varphi_{1} \wedge \cdots \wedge \widehat{\varphi}_{i} \wedge \cdots \wedge \widehat{\varphi_{j}} \wedge \cdots \wedge \varphi_{k} \otimes \sigma .
\end{aligned}
$$

Now we have all ingredients at hand to compute the action of the curved Casimir on a tractor bundle.

Proposition 4. Let $V$ be a finite dimensional irreducible representation of $G$, and let $c_{0}$ be the scalar by which the Casimir operator acts on $V$. Let $(p: \mathcal{G} \rightarrow M, \omega)$ be a parabolic geometry of type $(G, P)$, let $V M \rightarrow M$ be the tractor bundle corresponding to $V$, and let $\nabla^{V}$ be the canonical tractor connection on this bundle.

Then the curved Casimir operator $\mathcal{C}: \Gamma(V M) \rightarrow \Gamma(V M)$ is given by $\mathcal{C}(\sigma)=c_{0}+2 \partial^{*} \nabla^{V} \sigma$.

Proof. It suffices to verify this locally over an open subset $U \subset M$ over which there is an adapted frame $\left\{X_{i}, A_{r}, Z^{i}\right\}$ for $\mathcal{A} M$, and use the formula from Proposition 3. Since we are working on a tractor bundle, we can expand

$$
-\left\{Z^{i}, X_{i}\right\} \bullet \sigma=-Z^{i} \bullet X_{i} \bullet \sigma+X_{i} \bullet Z^{i} \bullet \sigma .
$$


Using the relation between the tractor connection and the fundamental derivative, we conclude that $\left.\mathcal{C}(\sigma)\right|_{U}$ is given by

$$
-2 \sum_{i} Z^{i} \bullet \nabla_{\Pi\left(X_{i}\right)}^{V} \sigma+\sum_{i}\left(X_{i} \bullet Z^{i} \bullet \sigma+Z^{i} \bullet X_{i} \bullet \sigma\right)+\sum_{r} A^{r} \bullet A_{r} \bullet \sigma,
$$

where $\Pi: \mathcal{A} M \rightarrow T M$ is the natural projection. By construction, $\left\{\Pi\left(X_{i}\right)\right\}$ is a local frame for $T M$ over $U$ and $\left\{Z^{i}\right\}$ is the dual frame of $T^{*} M$, so the first sum in this formula gives $2 \partial^{*} \nabla^{V} \sigma$. The rest of the formula is tensorial, with the elements only acting on the values of $\sigma$. But on the values, we simply obtain the iterated actions of the elements of dual bases of $\mathfrak{g}$, so this produces the action of the Casimir on $V$.

\subsection{Tractor bundle valued forms}

The canonical connection $\nabla^{V}$ naturally extends to the covariant exterior derivative $d^{\nabla^{V}}$ on tractor bundle valued differential forms. The definition of $d^{\nabla^{V}}: \Omega^{k}(M, V M) \rightarrow \Omega^{k+1}(M, V M)$ is obtained by replacing in the standard formula for the exterior derivative the actions of vector


by the action of the curvature $R^{V}$ of $\nabla^{V}$. For a tractor connection, this curvature is induced by the curvature of the canonical Cartan connection, see Proposition 2.9 of [5]. In particular, for the homogeneous model, $d^{\nabla^{V}} \circ d^{\nabla^{V}}=0$, and $\left(\Omega^{*}\left(G / P, G \times_{P} V\right), d^{\nabla^{V}}\right)$ is a fine resolution of the constant sheaf $V$ on $G / P$. This is called the twisted de-Rham resolution.

On the other hand, there is a natural resolution of the finite dimensional representation $V$ by generalized Verma modules. This is the (generalized) Bernstein-Gelfand-Gelfand (BGG) resolution, constructed in [1] for the Borel subgroup and in [15] in general. The construction of these resolutions is purely algebraic, using existence results for homomorphisms between generalized Verma modules, and combinatorial properties of Weyl groups. Now there is a duality between homomorphisms of generalized Verma modules and invariant differential operators between sections of homogeneous bundles, see appendix A of the preprint version of [6] for an exposition. Via this duality, the sequence of homomorphisms forming the BGG resolution corresponds to a sequence of invariant differential operators on sections of homogeneous bundles over $G / P$.

As mentioned before, constructing invariant differential operators for parabolic geometries is an important and difficult problem. It is known that the BGG resolutions contain a majority of the existing homomorphisms between generalized Verma modules, so trying to obtain the corresponding operators for curved geometries is a natural idea. This problem was solved in [6] and the construction was significantly improved in [3]. The idea is to construct the BGG operators from the twisted de-Rham resolution in a way which extends to the curved case. There the composition of two covariant exterior derivatives is non-zero, so the twisted de-Rham sequence is not a complex any more. Hence one only obtains a sequence of invariant differential operators, called a BGG-sequence. It should be mentioned however, that under certain semiflatness assumptions, curved BGG sequences may contain very interesting subcomplexes, see [7].

The relation between the two sequences is provided by Lie algebra homology. Let $(p: \mathcal{G} \rightarrow$ $M, \omega)$ be a parabolic geometry of type $(G, P)$, let $V$ be a representation of $G$ and consider the operators $\partial^{*}$ acting on $\Lambda^{k} T^{*} M \otimes V M$ as defined in Section 4.1. It is well known that $\partial^{*} \circ \partial^{*}=0$, so $\operatorname{im}\left(\partial^{*}\right) \subset \operatorname{ker}\left(\partial^{*}\right) \subset \Lambda^{k} T^{*} M \otimes V M$ are natural subbundles. The subquotient $\operatorname{ker}\left(\partial^{*}\right) / \operatorname{im}\left(\partial^{*}\right)$ is denoted by $H_{k}\left(T^{*} M, V M\right)$, since it the just the fiber-wise Lie algebra homology. By construction it is the associated bundle $\mathcal{G} \times_{P} H_{k}\left(\mathfrak{p}_{+}, V\right)$. A crucial fact (see [6]) is that the natural action of $\mathfrak{p}_{+}$on $\Lambda^{k} \mathfrak{p}_{+} \otimes V$ maps $\operatorname{ker}\left(\partial^{*}\right)$ to $\operatorname{im}\left(\partial^{*}\right)$. Hence $\mathfrak{p}_{+}$acts trivially on the homology $H_{k}\left(\mathfrak{p}_{+}, V\right)$, so this is a completely reducible representation.

All this also works for a general representation $W$, but then it is not clear what the homology groups look like. However, for a representation $V$ of $\mathfrak{g}$, the $\mathfrak{g}_{0}$-representations $H_{k}\left(\mathfrak{p}_{+}, V\right)$ are 
computed by Kostant's version of the Bott-Borel-Weil theorem proved in [14]. Kostant actually computes the Lie algebra cohomology groups $H^{k}\left(\mathfrak{p}_{+}, V\right)$, but they are dual to the homology groups. The description is algorithmic, so the computation can be easily carried out explicitly. The result is that these are exactly the duals of the representations showing up in the Vermamodule version of the BGG resolution, so they are exactly the representations we need.

By construction, for each $k$ and the subbundle $\operatorname{ker}\left(\partial^{*}\right) \subset \Lambda^{k} T^{*} M \otimes V M$, we have a natural bundle projection $\pi_{H}: \operatorname{ker}\left(\partial^{*}\right) \rightarrow H_{k}(M, V M)$. This induces a tensorial operator on sections, which we denote by the same symbol. The crucial step in the construction of BGG sequences is to construct invariant splitting operators $L: \Gamma\left(H_{k}(M, V M)\right) \rightarrow \Gamma\left(\operatorname{ker}\left(\partial^{*}\right)\right)$ such that $\pi_{H} \circ L=\mathrm{id}$. This is done by looking at $\square^{R}:=d^{\nabla^{V}} \circ \partial^{*}+\partial^{*} \circ d^{\nabla^{V}}$, which is an invariant first order operator defined on $\Omega^{k}(M, V M)$. By construction, $\square^{R}$ commutes with $\partial^{*}$ and hence preserves sections of the natural subbundles $\operatorname{ker}\left(\partial^{*}\right)$ and $\operatorname{im}\left(\partial^{*}\right)$. Further, it turns out that, similarly as discussed for the adjoint tractor bundle in Section 3.2, any tractor bundle is canonically filtered. The operator $\square^{R}$ is filtration preserving, i.e. maps sections of any filtration component to sections of the same component.

Hence one can look at the lowest homogeneous component, or otherwise put, the induced operator on the associated graded vector bundle. This turns out to be tensorial and induced by the Kostant-Laplacian $\square$, which plays a crucial role in Kostant's proof in [14]. In particular, the action of $\square$ on sections of the subbundle $\operatorname{im}\left(\partial^{*}\right)$ is immediately seen to be invertible. From this, one concludes that $\square^{R}$ acts invertibly on $\Gamma\left(\operatorname{im}\left(\partial^{*}\right)\right)$, and the inverse $Q$ is a differential operator, which is invariant by construction. Then for $\sigma \in \Gamma\left(H_{k}(M, V M)\right)$ one can choose any $\varphi \in \Gamma\left(\operatorname{ker}\left(\partial^{*}\right)\right)$ such that $\pi_{H}(\varphi)=\sigma$ and define $L(\sigma):=\varphi-Q\left(\square^{R}(\varphi)\right)$. It turns out that $d^{\nabla^{V}} \circ L$ has values in $\operatorname{ker}\left(\partial^{*}\right)$, so one may define the BGG operators as $D^{V}:=\pi_{H} \circ d^{\nabla^{V}} \circ L$. Finally, for $\varphi \in \Gamma\left(\operatorname{ker}\left(\partial^{*}\right)\right)$, vanishing of $\partial^{*} d^{\nabla^{V}} \varphi$ already implies vanishing of $\square^{R}(\varphi)$ and hence $\varphi=L\left(\pi_{H}(\varphi)\right)$, which is a very useful characterization of the splitting operator.

\subsection{The relation to the curved Casimir}

We next discuss the relation of the operator $\square^{R}=\partial^{*} \circ d^{\nabla^{V}}+d^{\nabla^{V}} \circ \partial^{*}$ to the curved Casimir operator. We will get a complete description in the locally flat case and a large amount of information in general. The main tool for these results is the following lemma which holds for general geometries.

Lemma 2. Let $(p: \mathcal{G} \rightarrow M, \omega)$ be a parabolic geometry of type $(G, P), V$ an irreducible representation of $G$, and $V M$ the corresponding tractor bundle. Let $c_{0} \in \mathbb{R}$ be the eigenvalue of the Casimir operator of $\mathfrak{g}$ on the irreducible representation $V$.

Then the action of the curved Casimir operator $\mathcal{C}$ on $\Omega^{k}(M, V M)$ is compatible with the natural filtration on $\Lambda^{k} T^{*} M \otimes V M$ discussed in Section 4.2 and the induced action on the sections of the associated graded vector bundle is given by $2 \square+c_{0}$, where $\square$ denotes the bundle map induced by the Kostant-Laplacian.

Proof. The filtration of $\Lambda^{k} T^{*} M \otimes V M$ is formed by natural subbundles, so the fact that sections of these subbundles are preserved by $\mathcal{C}$ follows immediately from naturality as proved in Proposition 2. The associated graded vector bundle is induced by a representation with trivial action of $\mathfrak{p}_{+}$and diagonalizable action of $\mathfrak{z}\left(\mathfrak{g}_{0}\right)$, so it splits into a direct sum of bundles induced by irreducible representations of $P$. Again by naturality, the operator induced on the associated graded by $\mathcal{C}$ coincides with the curved Casimir on the bundle in question.

Now the proof of Theorem 1 shows that the latter curved Casimir acts by a scalar on each lowest weight vector, and hence on each isotypical component. Using the conventions of Section 3.4 on weights, this scalar is explicitly given by $\left\|\nu+\rho^{2}\right\|-\|\rho\|^{2}$ on the bundle corresponding to the isotypical component of lowest weight $-\nu$. 
Now it is a crucial part of Kostant's proof in [14], that the Kostant-Laplacian acts by a scalar on each isotypical component. Carrying out the dualizations needed to translate from Kostant's setting (cohomology of $\mathfrak{p}_{+}$) to the one we need here (homology of $\mathfrak{p}_{+}$with coefficients in the dual representation), this scalar is given by $\frac{1}{2}\left(\|\nu+\rho\|^{2}-\|\lambda+\rho\|^{2}\right)$ on the isotypical component of weight $-\nu$, where $-\lambda$ is the lowest weight of $V$. But then the claim follows immediately since if $-\lambda$ is the lowest weight of $V$, then $c_{0}=\left\|\lambda+\rho^{2}\right\|-\|\rho\|^{2}$.

On the one hand, this result immediately implies that the operator $\mathcal{C}-c_{0}$ on $\Omega^{k}(M, V M)$ is filtration preserving and induces $2 \square$ on the associated graded. Hence one can construct curved BGG sequences replacing the operator $\square^{R}$ by $\mathcal{C}-c_{0}$. There is not much difference between these two operators, however, because we have the following result.

Proposition 5. On any parabolic geometry $(p: \mathcal{G} \rightarrow M, \omega)$ of type $(G, P)$ and any tractor bundle $V M$, the operator $2 \square^{R}-\mathcal{C}+c_{0}$ on $\Omega^{k}(M, V M)$ is tensorial and maps each component of the natural filtration of $\Lambda^{k} T^{*} M \otimes V M$ to the next smaller filtration component.

Proof. Since both $2 \square^{R}$ and $\mathcal{C}-c_{0}$ are filtration preserving, so is their difference. But by Lemma 2, this difference induces the zero map on the associated graded bundle, and hence moves each filtration component to the next smaller one. So it remains to prove that the difference is tensorial, i.e. linear over $C^{\infty}(M, \mathbb{R})$.

Now $\partial^{*}$ is tensorial, and for $d^{\nabla^{V}}$ we have the Leibniz rule $d^{\nabla^{V}}(f \varphi)=d f \wedge \varphi+f d^{\nabla^{V}} \varphi$. This implies that

$$
\square^{R}(f \varphi)=f \square^{R}(\varphi)+\partial^{*}(d f \wedge \varphi)+d f \wedge \partial^{*} \varphi=f \square^{R}(\varphi)-d f \bullet \varphi,
$$

where for the last step we used formula (1.2) of [6].

On the other hand, the fundamental derivative satisfies a Leibniz rule by Proposition 3.1 of [5] and naturality. Moreover, on smooth functions, the fundamental derivative coincides with the exterior derivative and the action map $\bullet$ is trivial. Using these facts, the formula for $\mathcal{C}$ in terms of adapted local frames from Proposition 3 immediately implies that

$$
\mathcal{C}(f \varphi)=f \mathcal{C}(\varphi)-2 \sum_{i} d f\left(X_{i}\right) Z^{i} \bullet \varphi=f \mathcal{C}(\varphi)-2 d f \bullet \varphi .
$$

Together with the above, this shows that $2 \square^{R}-\mathcal{C}+c_{0}$ is tensorial, which completes the proof.

Using this result, we can completely clarify the situation on the homogeneous model, and hence for locally flat geometries (which are locally isomorphic to the homogeneous model).

Corollary 1. Suppose that $V$ is an irreducible representation of $\mathfrak{g}$ and let $c_{0}$ be the eigenvalue of the Casimir operator on $V$. Then on the homogeneous model and hence on locally flat geometries, the curved Casimir operator on differential forms with values in the tractor bundle induced by $V$ is given by $\mathcal{C}=2 \square^{R}+c_{0}$.

Proof. From Proposition 5 above we know that for each $k$, the operator $2 \square^{R}+c_{0}-\mathcal{C}$ is a tensorial invariant operator on $G \times{ }_{P}\left(\Lambda^{k} \mathfrak{p}_{+} \otimes V\right)$, so this is induced by a $P$-equivariant linear map on $\Lambda^{k} \mathfrak{p}_{+} \otimes V$. In addition, the proposition tells us that this linear map has to map each of the components of the natural $P$-invariant filtration of $\Lambda^{k} \mathfrak{p}_{+} \otimes V$ into the next smaller filtration component. But it is well known that there is an element $A \in \mathfrak{g}_{0}$ (called the grading element) which acts diagonalizably on $\Lambda^{k} \mathfrak{p}_{+} \otimes V$ in such a way that the eigenvalues are of the form $a_{0}, a_{0}+1, \ldots, a_{0}+N$ and the filtration components are just the sum of the eigenspaces for the eigenvalues $a_{0}+i, \ldots, a_{0}+N$ for some $i$. But then equivariancy of our map for the action of $A$ implies that it is identically zero. 


\subsection{An interpretation of the BGG-resolution}

Let $V$ be an irreducible representation of $\mathfrak{g}$ and consider the twisted de-Rham resolution $\left(\Omega^{*}\left(G / P, G \times{ }_{P} V\right), d^{\nabla^{V}}\right)$ discussed in Section 4.2 on the homogeneous model. In terms of representation theory, this is a resolution of the locally constant sheaf $V$ on $G / P$ by induced representations and $G$-equivariant (differential) operators. On each of the spaces $\Omega^{k}\left(G / P, G \times_{P} V\right)$ we have the Casimir operator $\mathcal{C}=\mathcal{C}_{k}$, and naturality of the Casimir implies that $d^{\nabla^{V}} \circ \mathcal{C}_{k}=$ $\mathcal{C}_{k+1} \circ d^{\nabla^{V}}$. In particular, the operators $d^{\nabla^{V}}$ map eigenspaces of the Casimir operator $\mathcal{C}$ to eigenspaces for the same eigenvalue. The relevant eigenvalue here of course is the eigenvalue $c_{0}$ on the representation $V$, which is isomorphic to the space of sections of $G \times{ }_{P} V$ which are parallel for $\nabla^{V}$. Using known results on BGG sequences, we can determine the eigenspace of the Casimir for the eigenvalue $c_{0}$.

Proposition 6. The projection $\pi_{H}: \Omega^{k}\left(G / P, G \times{ }_{P} V\right) \rightarrow \Gamma\left(G \times{ }_{P} H_{k}\left(\mathfrak{p}_{+}, V\right)\right)$ restricts to a linear isomorphism on the eigenspace of the Casimir operator $\mathcal{C}$ for the eigenvalue $c_{0}$. In particular, this eigenspace is a finite direct sum of (the spaces of all smooth vectors in) principal series representations.

Proof. From Corollary 1 we know that the $c_{0}$-eigenspace of $\mathcal{C}$ coincides with the kernel of $\square^{R}=\partial^{*} \circ d^{\nabla^{V}}+d^{\nabla^{V}} \circ \partial^{*}$. Now let $L: \Gamma\left(G \times{ }_{P} H_{k}\left(\mathfrak{p}_{+}, V\right)\right) \rightarrow \Omega^{k}\left(G / P, G \times{ }_{P} V\right)$ be the BGG splitting operator as described in Section 4.2. Then $\pi_{H} \circ L=\mathrm{id}$, so $\pi_{H}$ is surjective. We have noted in Section 4.2 that $\partial^{*} \circ L=0$ and $\partial^{*} \circ d^{\nabla^{V}} \circ L=0$, and hence $L$ has values in $\operatorname{ker}\left(\square^{R}\right)$.

Conversely, assume that $\square^{R}(\varphi)=0$. By construction, $\square^{R}$ commutes with $\partial^{*}$, so this implies $\square^{R}\left(\partial^{*} \varphi\right)=0$. But we have noted in Section 4.2 that $\square^{R}$ acts invertibly on sections of $\operatorname{im}\left(\partial^{*}\right)$. Hence $\partial^{*} \varphi=0$ and we can form $\pi_{H}(\varphi)$. But since $\partial^{*} \varphi=0$, we get $0=\square^{R}(\varphi)=\partial^{*} d^{\nabla^{V}} \varphi$, and in Section 4.2 we have noted that these facts imply that $\varphi=L\left(\pi_{H}(\varphi)\right)$. Hence we conclude that $\pi_{H}$ and $L$ restrict to inverse isomorphisms between $\operatorname{ker}\left(\square^{R}\right)$ and $\Gamma\left(G \times_{P} H_{k}\left(\mathfrak{p}_{+}, V\right)\right)$.

In Theorem 2.6 of [6] it is shown that on the homogeneous model, any BGG sequence is a complex, which defines a fine resolution of the finite dimensional representation $V$. Here we obtain an alternative proof of this fact as well as a nice interpretation in terms of representations theory.

Corollary 2. Restricting the twisted de-Rham resolution $\left(\Omega^{*}\left(G / P, G \times_{P} V\right), d^{\nabla^{V}}\right)$ to the eigenspaces of the Casimir operator for the eigenvalue $c_{0}$ induces a finite resolution of the representation $V$ by direct sums of (spaces of smooth vectors in) principal series representations. The inducing P-representations for these principal series representations are the duals of the representations inducing the generalized Verma modules in the BGG resolution.

Proof. It is an elementary exercise to verify that the restriction of the twisted de-Rham resolution to eigenspaces for one fixed eigenvalue of the Casimir is again a resolution of the eigenspace in the kernel of the first operator for the given eigenvalue. Choosing the eigenvalue to be $c_{0}$, this eigenspace is (isomorphic to) $V$. By Proposition 6, the $c_{0}$-eigenspace in each $\Omega^{k}\left(G / P, G \times{ }_{P} V\right)$ is a direct sum as required. The inducing representations are described by Kostant's version of the Bott-Borel-Weil theorem, which proves the last claim.

\section{Acknowledgments}

The idea to study the Casimir operator on tractor bundle valued forms grew out of questions by M. Cowling and by P. Julg, who conjectured Corollary 1 for the case of the trivial representation. We are very grateful to them for drawing our attention to this problem. Our thanks also go to the anonymous referees for helpful suggestions and corrections. Most of the work was done during meetings of authors at the Erwin Schrödinger Institute for Mathematical Physics 
in Vienna. First author supported by project P19500-N13 of the Fonds zur Förderung der wissenschaftlichen Forschung (FWF). The second author thanks the grant GAČR Nr. 201/05/2117 and the institutional grant MSM 0021620839 for their support.

\section{References}

[1] Bernstein I.N., Gelfand I.M., Gelfand S.I., Differential operators on the base affine space and a study of $\mathfrak{g}$-modules, in Lie Groups and their Representations, Editor I.M. Gelfand, Adam Hilger, 1975, 21-64.

[2] Biquard O., Métriques d'Einstein asymptotiquement symétriques, Astérisque, Vol. 265, 2000.

[3] Calderbank D.M.J., Diemer T., Differential invariants and curved Bernstein-Gelfand-Gelfand sequences, J. Reine Angew. Math. 537 (2001), 67-103, math.DG/0001158.

[4] Čap A., Two constructions with parabolic geometries, Rend. Circ. Mat. Palermo Suppl. ser. II 79 (2006), 11-37, math.DG/0504389.

[5] Čap A., Gover A.R., Tractor calculi for parabolic geometries, Trans. Amer. Math. Soc. 354 (2002), 15111548.

[6] Čap A., Slovák J., Souček V., Bernstein-Gelfand-Gelfand sequences, Ann. of Math. 154 (2001), 97-113. Preprint version math.DG/0001164, 45 pages.

[7] Čap A., Souček V., Subcomplexes in Curved BGG Sequences, math.DG/0508534.

[8] Chern S.S., Moser J., Real hypersurfaces in complex manifolds, Acta Math. 133 (1974), 219-271.

[9] Eastwood M.G., Rice J.W., Conformally invariant differential operators on Minkowski space and their curved analogues, Comm. Math. Phys. 109 (1987), 207-228.

[10] Gover A.R., Graham C.R., CR invariant powers of the sub-Laplacian, J. Reine Angew. Math. 583 (2005), 1-27, math.DG/0301092.

[11] Gover A.R., Hirachi K., Conformally invariant powers of the Laplacian - a complete nonexistence theorem, J. Amer. Math. Soc. 17 (2004), 389-405, math.DG/0304082.

[12] Graham C.R., Conformally invariant powers of the Laplacian. II. Nonexistence, J. London Math. Soc. 46 (1992), 566-576.

[13] Graham C.R., Jenne R., Mason L.J., Sparling G.A., Conformally invariant powers of the Laplacian. I. Existence, J. London Math. Soc. 46 (1992), 557-565.

[14] Kostant B., Lie algebra cohomology and the generalized Borel-Weil theorem, Ann. of Math. 74 (1961), 329-387.

[15] Lepowsky J., A generalization of the Bernstein-Gelfand-Gelfand resolution, J. Algebra 49 (1977), 496-511.

[16] Sharpe R.W., Differential geometry, Graduate Texts in Mathematics, Vol. 166, Springer-Verlag, 1997.

[17] Tanaka N., On the equivalence problem associated with simple graded Lie algebras, Hokkaido Math. J. 8 (1979), 23-84.

[18] Yamaguchi K., Differential systems associated with simple graded Lie algebras, Adv. Stud. Pure Math. 22 (1993), 413-494. 